\title{
LISA Pathfinder
}

\section{P McNamara ${ }^{1}$, S Vitale ${ }^{2}$ and K Danzmann ${ }^{3}$ (on behalf of the LISA Pathfinder Science Working Team)}

${ }^{1}$ European Space Agency, Keplerlaan 1, 2201 AG Noordwijk, The Netherlands

${ }^{2}$ Department of Physics, University of Trento, Via Sommarive 14, I-38100 Povo, Italy

3 Albert-Einstein-Institute and University of Hannover, 30167 Hannover, Germany

Received 15 November 2007, in final form 8 January 2008

Published 15 May 2008

Online at stacks.iop.org/CQG/25/114034

\begin{abstract}
LISA Pathfinder (formerly known as SMART-2) is an ESA mission designed to pave the way for the joint ESA/NASA Laser Interferometer Space Antenna (LISA) mission by testing in-flight the critical technologies required for spaceborne gravitational wave detection; it will put two test masses in a nearperfect gravitational free fall, and control and measure their motion with an unprecedented accuracy. This is achieved through technology comprising inertial sensors, high-precision laser metrology, drag-free control and an ultraprecise micro-Newton propulsion system. The LISA Pathfinder mission is now in Phase C/D - the Implementation Phase, and is due to be launched in 2010, with results on the performance of the system being available within 6 months thereafter.
\end{abstract}

PACS numbers: $\quad$ 04.80.Nn, 95.30.Sf, 95.55.Ym

(Some figures in this article are in colour only in the electronic version)

\section{Introduction}

LISA Pathfinder (LPF), the second of the ESA Small Missions for Advanced Research in Technology (SMART), is a dedicated technology demonstrator for the joint ESA/NASA Laser Interferometer Space Antenna (LISA) mission [1].

LISA, a joint ESA/NASA mission to observe low-frequency gravitational waves, has continually been ranked as one of the most scientifically important missions under study [2]. However, the very concept of low-frequency gravitational wave detection, i.e. that a particle falling under the influence of gravity alone follows a geodesic in spacetime, has never been demonstrated to the required precision. This is the most basic assumption of Einstein's general relativity; LISA Pathfinder has been designed to test this hypothesis with an unprecedented accuracy.

The LISA Pathfinder mission essentially mimics one arm of the LISA constellation by shrinking the 5 million kilometre armlength down to a few tens of centimetres. The distance 
between the two test masses is measured using a laser interferometric technique similar to the proof-mass interferometer of the LISA interferometry system.

LISA Pathfinder is due to be launched in 2010 on-board a dedicated launch vehicle. The spacecraft and expendable propulsion module are injected into a low-Earth orbit $(200 \mathrm{~km}$ $\times 900 \mathrm{~km}$ ), from which, after a series of apogee raising manoeuvres, will enter a transfer orbit towards the first Sun-Earth Lagrange point (L1). After separation from the propulsion module, the LPF spacecraft will be stabilized using the micro-Newton thrusters, entering a Lissajous orbit around L1 (500 $000 \mathrm{~km}$ by $800000 \mathrm{~km}$ orbit).

Following the initial on-orbit check-out and instrument calibration, the in-flight demonstration of the LISA technology will take place. The nominal lifetime of the science operations is 180 days; this includes the LISA Technology Package (LTP) operations, the Disturbance Reduction System (DRS) operations and a period of joint operations when the LTP will control the DRS thrusters.

\section{LISA Pathfinder mission concept}

LISA Pathfinder will test in a space experiment that free falling bodies do follow geodesics in spacetime by more than two orders of magnitude better than any past or present mission (with the exception of LISA itself). The concept that a particle falling under the influence of gravity alone follows a geodesic in spacetime is at the very foundation of general relativity (GR).

In GR, gravity is not considered as an external force: instead gravity is the source of the spacetime curvature. Therefore, in a universe devolved of mass (a flat spacetime) free-falling test masses will move in straight lines with uniform velocity (Newton's 1st law). In the real universe (as described by general relativity), the presence of mass, hence gravity/curvature, modifies Newton's 1st law to state that in the absence of any external force, test masses move along geodesics.

All experiments aimed at directly measuring curvature caused by celestial bodies, or to test subtle effects of GR, e.g., frame dragging, detection of gravitational waves or to probe its very foundation - the equivalence principle, invariably search for violation of geodesic motion.

The difficulty of achieving 'high-purity geodesic motion' is that any parasitic forces compete with spacetime geometry to set masses into motion, perturbing them away from their geodesic lines. As gravity is by far the weakest of all fundamental interactions, achieving the required extremely low level of non-gravitational acceleration requires the understanding, reduction and control of the disturbances produced by a wide range of physical phenomena.

LISA Pathfinder's experiment concept is to improve the uncertainty in the proof of geodesic motion. This is achieved by tracking, using pico-metre resolution laser interferometry, two test masses nominally in free fall (see figure 1) and by showing that their relative parasitic acceleration, at frequencies around $1 \mathrm{mHz}$, is at least two orders of magnitude smaller than anything demonstrated or planned so far. The LISA Pathfinder spacecraft as an inertial platform, free of spurious accelerations, will be the best laboratory ever created for fundamental physics experiments, where the conditions that Einstein could only think of in his gedankenexperiment, will be realized in the real world (see figure 1).

LISA Pathfinder is both a mission in general relativity and in precision metrology, pushing these disciplines several orders of magnitude beyond their current state-of-the-art. In doing so it opens new ground for an entire class of new missions in general relativity, in fundamental physics at large and in Earth observation. The high-resolution optical readout of test-mass motion allows test-mass to test-mass tracking even when they are located in different spacecraft, 


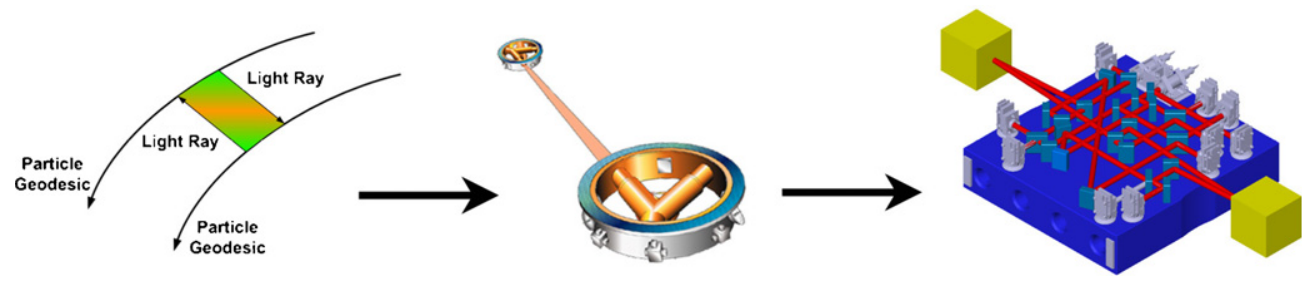

Figure 1. The classical Einstein gedankenexperiment (left), one arm of LISA showing the relationship to the gedankenexperiment (middle) and LISA technology package, showing the two free-floating test masses and the laser interferometer (right).

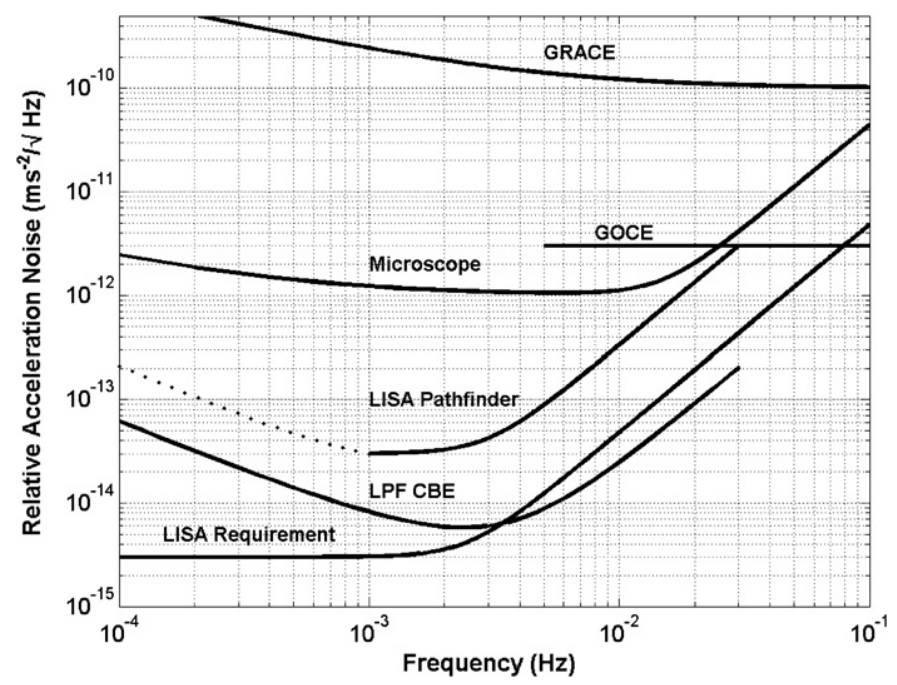

Figure 2. Comparison of the performance of several missions. The line labelled 'LPF CBE' is the current best estimate of the expected performance of LPF, the line labelled 'LISA Pathfinder' is the LPF science requirement.

at large distances and in interplanetary space, e.g. LISA, or at short distance in low-Earth orbit, like in future geodesy missions.

Figure 2 shows the comparison of the required performances of various missions for relative geodesic deviation of the test mass pairs [3].

It must be stated that the true objective of LISA Pathfinder is not to develop hardware, but to confirm the overall physical model of the forces that act on a test mass in the interplanetary space. To fulfil this programme, the mission is not going to just make a measurement of acceleration but will implement a full menu of measurements: at the end of this set of measurements, the residual acceleration noise model will be verified down to painstaking detail.

\section{Performance goals}

The mission goals of the LTP can be split into three covering the performance of the inertial sensor, the performance of the laser interferometer and aspects of the flight readiness of the 
technologies critical for a successful LISA mission. The mission goals can be summarized as follows (for the full mission performance requirements, please refer to the LISA technology package science requirements document [4]).

- Demonstrate that a test mass can be put in pure gravitational free fall within one order of magnitude of the requirement for LISA. The one order of magnitude rule applies also to frequency; thus the flight test of the LTP on LPF is considered satisfactory if free fall of one TM is demonstrated to be within

$$
S_{a}^{1 / 2}(f) \leqslant 3 x \times 10^{-14}\left[1+\left(\frac{f}{3 \mathrm{mHz}}\right)^{2}\right] \mathrm{ms}^{-2} \mathrm{~Hz}^{-1 / 2}
$$

over the frequency range, $f$, of 1 to $30 \mathrm{mHz}$.

- Demonstrate laser interferometry with free-falling mirrors (test masses of the LTP) with displacement sensitivity meeting the LISA requirements over the LTP measurement bandwidth. Thus, the flight test of LTP is considered satisfactory if the laser metrology resolution is demonstrated to be within

$$
S_{\delta x}^{1 / 2}(f)=9.1 \times 10^{-12}\left[1+\left(\frac{f}{3 \mathrm{mHz}}\right)^{-2}\right] \mathrm{mHz}^{-1 / 2}
$$

over the frequency range, $f$, of 1 to $30 \mathrm{mHz}$.

- Assess the lifetime and reliability of the micro-Newton thrusters, lasers and optics in a space environment.

\section{Technology}

The goal of LTP is to develop hardware that, with only minor changes, can be directly exploited in the development of LISA.

The laser of LTP is a Nd:YAG non-planar ring oscillator (NPRO) manufactured by Tesat $\mathrm{GmbH}$. The laser emits $\sim 30 \mathrm{~mW}$ of $1064 \mathrm{~nm}$ light through a single-mode, polarization maintaining fibre. This light is then split into two paths, with each path delivered to a separate acousto-optic modulator (AOM), manufactured by Contraves. The AOMs are used as the actuator for the intensity stabilization system and to frequency shift the light, resulting in a relative heterodyne frequency of approximately $2 \mathrm{kHz}$. The frequency-shifted beams are then delivered, again by a single-mode, polarization-maintaining fibre, to the LTP core assembly (see figure 3), specifically to the optical bench. The optical bench (manufactured by the University of Glasgow) consists of a Zerodur base plate, with fused silica optics hydroxycatalysis bonded (silicate bonded) to the surface. One of the laser beams delivered to the optical bench is used as a reference beam. The other beam is used for the measurement of the position (and hence acceleration) of the test masses. In total, six different measurements are made on the bench, four heterodyne measurements, namely, relative displacement of the test masses $\left(x_{2}-x_{1}\right)$, position of test mass one with respect to the optics bench $\left(x_{1}\right)$, frequency noise and a reference interferometer; and two measurements consisting of the measurement beam and reference beam intensities (see right hand drawing in figure 1). The heterodyne signals are detected via quadrant photodiodes, and the phase of the beat signal is measured using a dedicated phase measurement system (designed by the Albert Einstein Institute, Hannover, and manufactured by the University of Birmingham).

The main goal of the LTP is to demonstrate that a body can be put into a near-perfect free fall. A demonstration of the technology required to meet this requirement is the main driver for the LPF mission. The inertial sensor, designed by the University of Trento, is a subsystem 


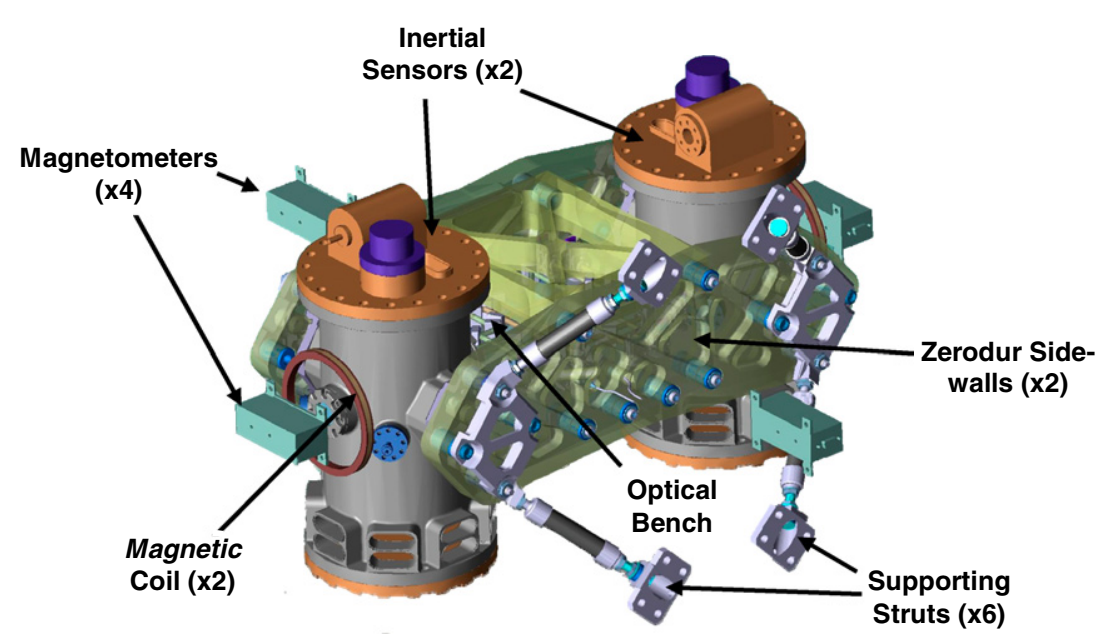

Figure 3. CAD drawing of the LISA technology package core assembly.

designed such that a cubic test mass, located at the centre, is free (as far as possible) from all external forces bar inertial gravity. The system consists of: a gold-platinum test mass of $46 \mathrm{~mm}$ on a side (manufactured by Heraeus); a capacitive sensing and actuation system comprising the electrode housing (Laben) and front-end electronics (Contraves), a charge management system (Imperial College London), a caging mechanism (Laben) and finally a vacuum enclosure and equipment to maintain an ultra-high vacuum around the test mass (Carlo Gavazzi Space). The integration of the inertial sensor is the responsibility of Carlo Gavazzi Space. Photographs of the inertial sensor hardware, along with a cross-section of the complete system are shown in figure 4.

The position of the test mass is sensed by the laser interferometer in the sensitive axis and by the capacitive sensors in the non-sensitive axes. As the sensitive axes of the LTP masses are co-linear (as opposed to $60^{\circ}$ to each other in LISA), it is not possible for both masses to be drag free in their sensitive axes at the same time. In the nominal operational mode, one mass $\left(\mathrm{TM}_{1}\right)$ is used as the reference and the second mass is forced to follow (at a frequency below the measurement bandwidth) using electrostatic forcing applied via the capacitive plates. The control of the spacecraft around the drag-free mass, as well as the control of the second test mass, is performed by the drag-free and attitude control system (DFACS), designed by Astrium $\mathrm{GmbH}$.

The attitude of the spacecraft is controlled via a set of micro-Newton thrusters. LPF will carry two sets of such thrusters onboard, namely, field emission electric propulsion (FEEP) and US provided colloidal thrusters. The FEEP thrusters have a maximum thrust of $\sim 150 \mu \mathrm{N}$, with a resolution of $0.1 \mu \mathrm{N}$, while the colloids have the same resolution, but a thrust range of only up to $30 \mu \mathrm{N}$.

One other essential ingredient to the success of the LTP is the data diagnostic system (DDS) provided by IEEC, Spain. This subsystem consists of environmental monitoring hardware (thermometers, magnetometers) and environmental stimuli (heaters, coils). During the operation of the LTP, these systems will be used to measure transfer functions and cross couplings affecting the system, and make possible, among others, the extrapolation of the data to the LISA specific case. 


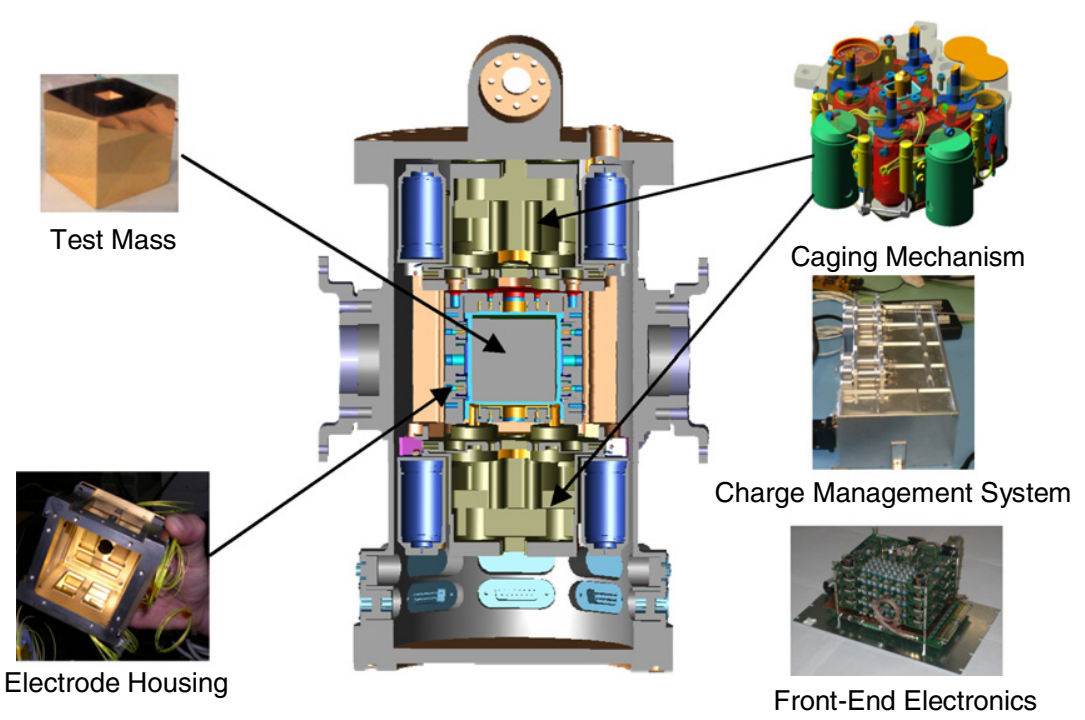

Figure 4. Inertial sensor system. The middle graphic shows a cross-section of the inertial sensor housed within the vacuum enclosure. The photographs and drawings around the edge show the engineering models of specific hardware subsystems, namely, the test mass, electrode housing, caging mechanism, charge management system (photograph shows the UV lamp unit) and frontend electronics.

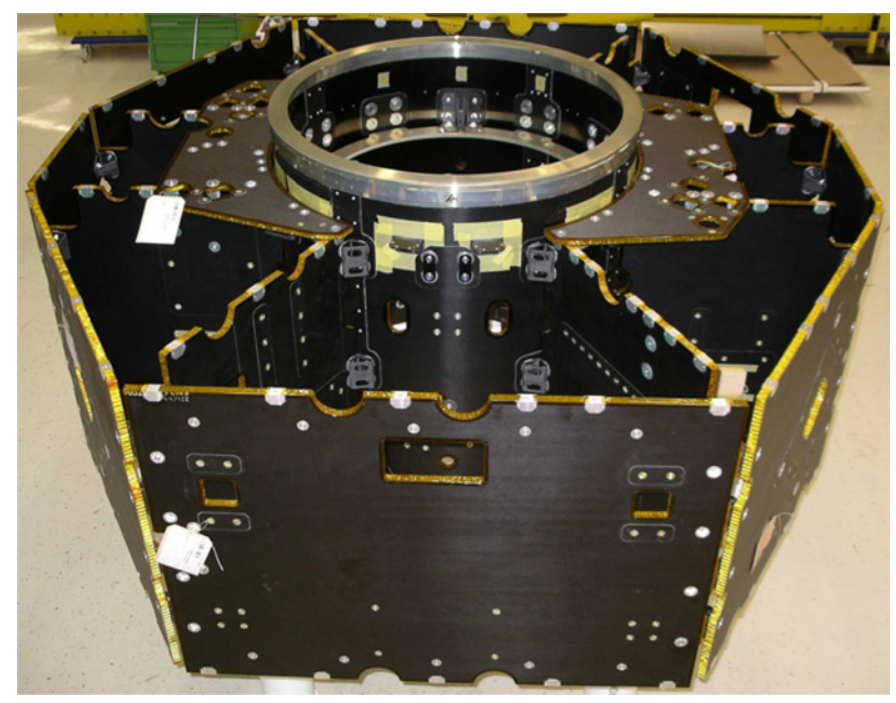

Figure 5. Flight structure of the LISA Pathfinder spacecraft.

\section{Mission status}

The LPF mission is now in Phase C/D: implementation phase. The spacecraft structure (figure 5) has been delivered to the LPF prime contractor, and is currently undergoing static load testing. Several hardware subsystems have also been delivered and will be integrated onto the spacecraft in due course. 
The LTP has undergone the Critical Design Review (CDR) in October 2007, with the System CDR expected in September 2008.

\section{Experiment timeline}

The philosophy behind the LTP mission planning is to conservatively work towards the mission goal of demonstrating the science requirements [5]. As with all satellite-based missions, as well as ground-based experiments, failures can occur which prematurely end the mission. Therefore, the LTP science operations are being designed such that the maximum information is gained from each stage of the mission, with riskier operations being performed towards the end of the mission.

The science operations can be split into the following phases.

- Pre-commissioning

- Advanced commissioning

- Noise identification and noise linear spectral density (LSD) minimization

- Noise model detailed investigation

- Extended investigation

- Extended mission

The pre-commissioning phase starts while the spacecraft is in transfer to its final orbit. This phase essentially tests the functionality of the subsystems not involving the test mass, i.e. laser, photodiodes, etc.

Once in orbit around L1, the mission enters the advanced commissioning phase. The goal of this phase is to release the test masses and make the first measurement of the acceleration noise of the system. This is achieved sequentially, i.e. first the masses will be suspended electrostatically, and a noise measurement made, followed by the transition to drag-free/lowfrequency suspension with noise measurements being made at each step in the process. With the system in the drag-free mode, the diagnostic systems will be turned on and checked out.

The noise identification and LSD minimization phase aims to demonstrate the required performance goal of $3 \times 10^{-14} \mathrm{~ms}^{-2} \mathrm{~Hz}^{-1 / 2}$ over the measurement bandwidth. To achieve this objective, the main sources of noise are identified and, where possible, minimized. The optimized noise spectral density is then compared with the prediction obtained from the noise projections using the measured transfer functions and environmental parameter noise.

In the noise model investigation phase dedicated experiments are performed to identify the dominating sources of residual noise and to characterize important experiment parameters in detail. The main aim of this phase is a quantative demonstration of the noise model to allow extrapolation to LISA.

The extended investigation phase is a period of time dedicated to perform other key experiments that are part of LPF. The information gained from these experiments will be used in the design and development of the LISA mission, but is supplemental to the information obtained in the previous phases. Examples of experiments to be performed in this phase include long data runs (covering the frequency band down to the LISA lower bound of $30 \mu \mathrm{Hz}$ ) and continuous discharging of the test masses. There will also be time allocated to perform other experiments suggested by the science community through the LPF science team. 


\section{Conclusions}

LISA Pathfinder is now in Phase C/D. Both the spacecraft and payload have successfully passed the preliminary design review, and the mission is on track for a launch in 2010. Not only will LISA Pathfinder test in-flight the technologies required for LISA, but it has also allowed the engineering difficulties associated with such a complex mission to be discovered, and overcome, prior to the design and development of LISA. This, coupled with the lessons learned in the operation of the spacecraft and payload, paves the way for the future success of LISA.

\section{Acknowledgments}

The authors would like to thank the entire LISA Pathfinder team for their continued work in making this mission possible. The LISA Pathfinder mission is funded through the European Space Agency, while the LTP is funded by ESA, ASI, CNES, DLR, MEC-PNE, SRON, STFC and PRODEX.

\section{References}

[1] Bender P et al 1998 LISA Pre-Phase A Report, MPQ 208, Munich, unpublished

[2] National Research Council 2007 NASA's Beyond Einstein Program: An Architecture for Implementation (Washington, DC: National Academy Press)

[3] Vitale S et al 2007 LISA Pathfinder: Einstein's Geodesic Explorer ESA-SCI(2007)1

[4] Vitale S et al 2005 Science Requirements and Top-Level Architecture Definition for the LISA Technology Package (LTP) on board LISA Pathfinder (SMART-2), ESA Ref: LTPA-UTN-ScRD-Iss3-Rev1

[5] McNamara P W et al 2006 LISA Pathfinder Experiments Master Plan, ESA Ref: S2-EST-PL-5007 